University of Nebraska - Lincoln

DigitalCommons@University of Nebraska - Lincoln

November 1994

\title{
Sputtering pressure effects and temperature-dependent magnetism of Co/Pd multilayers
}

\author{
S.Y. Jeong \\ University of Nebraska - Lincoln \\ Z.S. Shan \\ University of Nebraska - Lincoln \\ P. He \\ University of Nebraska - Lincoln \\ J.X. Shen \\ University of Nebraska - Lincoln \\ Y.B. Zhang \\ University of Nebraska - Lincoln \\ See next page for additional authors
}

Follow this and additional works at: https://digitalcommons.unl.edu/physicssellmyer

Part of the Physics Commons

Jeong, S.Y.; Shan, Z.S.; He, P.; Shen, J.X.; Zhang, Y.B.; Woollam, John A.; and Sellmyer, David J., "Sputtering pressure effects and temperature-dependent magnetism of Co/Pd multilayers" (1994). David Sellmyer Publications. 102.

https://digitalcommons.unl.edu/physicssellmyer/102

This Article is brought to you for free and open access by the Research Papers in Physics and Astronomy at DigitalCommons@University of Nebraska - Lincoln. It has been accepted for inclusion in David Sellmyer Publications by an authorized administrator of DigitalCommons@University of Nebraska - Lincoln. 


\section{Authors}

S.Y. Jeong, Z.S. Shan, P. He, J.X. Shen, Y.B. Zhang, John A. Woollam, and David J. Sellmyer 


\title{
Sputtering pressure effects and temperature-dependent magnetism of $\mathrm{Co} / \mathrm{Pd}$ multilayers
}

\author{
S. Y. Jeong, a) Z. S. Shan, P. He, J. X. Shen, Y. B. Zhang, J. A. Woollam, and \\ D. J. Sellmyer \\ Behlen Laboratory of Physics and Center for Materials Research and Analysis, University of Nebraska, \\ Lincoln, Nebraska 68588-0113
}

The temperature dependence of the sputtering Ar pressure effects on magnetic properties and the coercivity mechanism of $\mathrm{Co}(2 \AA) / \mathrm{Pd}(13 \AA)$ multilayers were studied as the sputtering Ar pressure varied from 3-15 mTorr and the temperature from 300 to $35 \mathrm{~K}$. It is found that the roughness of the interfaces or film surface increases with increasing sputtering pressure, the anisotropy increases with decreasing temperalure and increasing Ar pressure and shows a maximum at $P_{\mathrm{Ar}} \approx 12 \mathrm{mTorr}$, and the coercivity increases with Ar pressure and shows stronger temperature dependence at higher Ar pressure. The coercivity mechanism was analyzed in terms of the coercivity predicted by Kronmüller's theory [Phys. Status Solidi B 144, 385 (1987)]. Wall pinning is found to be the main mechanism and the size of the pinning site increases slightly as the Ar pressure increases.

\section{INTRODUCTION}

$\mathrm{Co} / \mathrm{Pd}$ multilayers have been studied intensively in the last decade for pure and applied reasons..$^{1-3}$ For the Co/Pd multilayers with nanoscale Co layer, the interfacial magnetism, which is strongly influenced by the preparation conditions, plays a crucial role in determining the magnetic behavior. Hashimoto et al., ${ }^{4}$ de Haan et al., ${ }^{5}$ Shin et al. ${ }^{6}$ and He et $a l^{7}$ have reported the Ar pressure effects on magnetic properties at room temperature. It is found that the coercivity increases with increasing $\mathrm{Ar}$ pressure $P_{\mathrm{Ar}}$ during deposition and the anisotropy increases monotonically with increasing $P_{\mathrm{AT}}$ (up to $P_{\mathrm{Ar}} \simeq 56 \mathrm{mTorr}$ ), ${ }^{5}$ or shows a maximum at $P_{\mathrm{Ar}}=10$ mTorr. ${ }^{4,6}$

In this article the temperature dependence of the sputtering pressure effects on magnetism was studied as the temperature varied from 300 to $35 \mathrm{~K}$. The coercivity mechanism was investigated in terms of the initial magnetization curves and minor loops at different temperatures, and comparisons were made to Kronmüller's model. ${ }^{8}$

\section{EXPERIMENT}

$[\mathrm{Co}(2 \AA) / \operatorname{Pd}(13 \AA)] \times 35$ (35 is the number of bilayers) multilayers were deposited onto glass substrates by dc magnetron sputtering under pressure $P_{\mathrm{Ar}}=3,6,9,12$, and 15 mTorr. All five samples were fabricated in one vacuum run to insure identical preparation conditions except for the $\mathrm{Ar}$ pressure.

The structure properties were characterized with the $\mathrm{x}$-ray diffraction and atomic force microscopy (AFM) and the magnetic properties were measured by an alternating gradient force magnetometer (AGFM) with the temperature changed from 300 to $35 \mathrm{~K}$. The coercivity $H_{c}(T)$ and magnetization $M(T)$ data were obtained from the perpendicular hysteresis loops and the measured anisotropy $K_{u}^{\prime}(T)$ data were determined from the area between the parallel and perpendicular magnetization curves.

\footnotetext{
a) Permanent address: Department of Physics, Gyeongsang National University, Chinju 660-701, Korea.
}

\section{RESULTS AND DISCUSSIONS}

\section{A. Structure properties}

Figure 1 shows the small-angle $x$-ray-diffraction patterns. It is seen clearly that the amplitude of the diffraction peaks decreases with increasing sputtering Ar pressure and when the sputtering pressure is greater than 9 mTorr, the diffraction peaks become obscure. This is attributed to the roughness of the interfaces which increases as the sputtering pressure increases since the sputtered $\mathrm{Co}$ and $\mathrm{Pd}$ atoms experienced more collisions with $\mathrm{Ar}$ atoms and form larger clusters at the growing film surface.

Figure 2 shows the AFM pictures of samples sputtered at (a) $P_{\mathrm{Ar}}=3 \mathrm{~m}$ Torr and (b) $15 \mathrm{~m}$ Torr and it is found that the surface roughness in Fig. 2(b) is much larger than that in Fig. 2(a). If the surface roughness may be regarded as the accumulation of the roughness of all individual layers or interfaces, Fig. 2 indicates clearly that the interfaces have larger roughness when sputtered in the higher Ar pressure, which is consistent with the result in Fig. 1.

\section{B. Temperature character of pressure effects on magnetic properties}

The Ar pressure dependence of the anisotropy $K_{u}\left(K_{u}\right.$ $=K_{u}^{\prime}+2 \pi M_{s}^{2}$ ) as the temperature varied from 300 to $35 \mathrm{~K}$ is demonstrated in Fig. 3. It is seen that $K_{u}$ increases as the temperature decreases. As the pressure increases $K_{u}$ first increases, then decreases and shows a small peak at $P_{\mathrm{Ar}}=12$ $\mathrm{mT}$ for all temperatures. This behavior is qualitatively consistent with earlier work ${ }^{4,6}$ except that our peaks are rather small; $K_{u}$ shows larger Ar pressure dependence at lower temperature. The origin of such $K_{u}$ behavior is attributed to the interfacial magnetism which strongly depends on the polarization of Pd atoms at the interfaces ${ }^{9,10}$ and the morphology of interfaces. As the temperature decreases the induced $\mathrm{Pd}$ moment increases which enhances the $K_{u}$. Hashimoto and co-workers ${ }^{4}$ have explained qualitatively the behavior of $\mathrm{Ar}$ pressure dependence of $K_{u}$ in terms of the stress-induced anisotropy because the stress in the film changes from compressive to tensile as the Ar pressure increases. Recently Vic- 


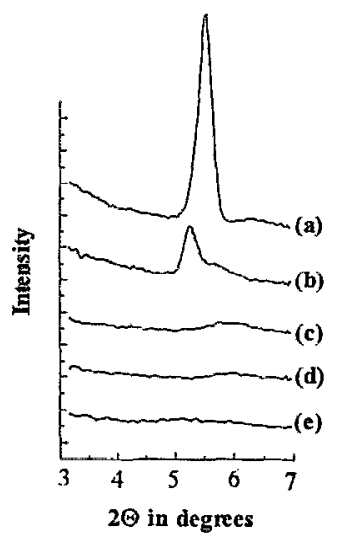

FIG. 1. Small-angle $\mathrm{x}$-ray diffraction for $\mathrm{Co}(2 \AA) / \operatorname{Pd}(13 \AA)$ deposited at different Ar sputtering pressures: (a) $3 \mathrm{mTorr}$; (b) $6 \mathrm{mTorr}$; (c) $9 \mathrm{mTorr}$; (d) 12 mTorr; and (e) 15 mTorr.

tora and MacLaren ${ }^{11}$ employed the symmetry-derived model based on summing $L(\mathbf{M} \cdot \mathbf{R})^{2}$ pair interactions (where $\mathbf{M}$ is the magnetization direction, $\mathbf{R}$ is the vector connecting the two atoms, and $L$ is an interaction parameter) to calculate anisotropy for $\mathrm{Co} / \mathrm{Pd}$ and $\mathrm{Co} / \mathrm{Pt}$ multilayers. We intend to use this approach to calculate the $K_{u}$ behavior quantitatively.

The sputtering pressure dependence of coercivity $H_{c}$ as the temperature varied from 300 to $35 \mathrm{~K}$ is shown in Fig. 4(a). The coercivity increases monotonically with increasing $P_{\text {Ar }}$ and shows stronger $P_{\mathrm{Ar}}$ dependence at the lower temperature. This behavior cannot be attributed fully to the change of $K_{u}$ as shown in Fig. 3. In order to understand such behavior properly, we also need to consider the pinning effect of the domain-wall motion which is discussed in more detail in the following section.

The temperature dependence of $H_{c}$ is shown in Fig. 4(b): $H_{c}$ increases as the temperature decreases and shows stron-
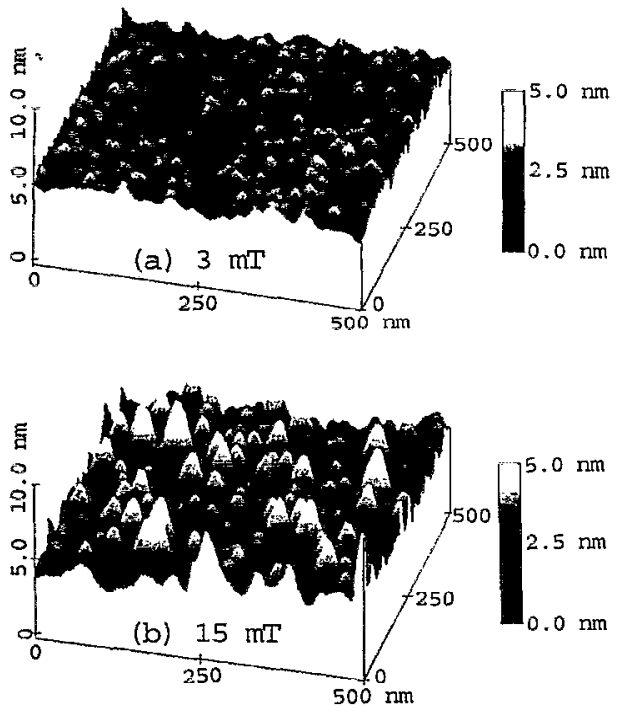

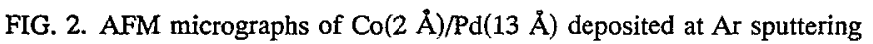
pressure of: (a) $3 \mathrm{~m}$ Torr and (b) $15 \mathrm{~m}$ Torr.

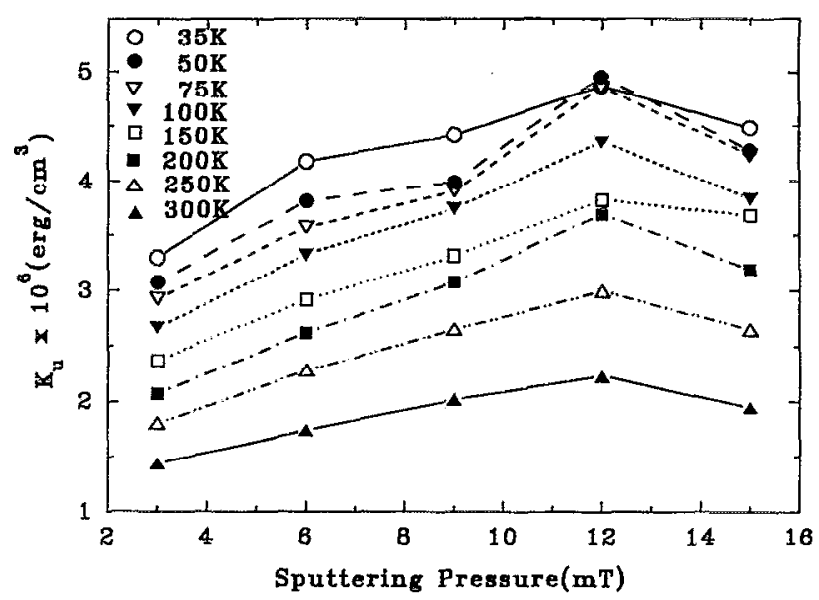

FIG. 3. Sputtering Ar pressure dependence of measured anisotropy $K_{u}^{\prime}$ at different temperatures.

ger temperature dependence at higher $P_{\mathrm{Ar}}$. The physical origins of this feature are discussed below.

\section{Coercivity mechanism}

In order to study the coercivity mechanism the initial curves and minor loops were measured at room and low temperature. All these curves show the typical domain-wall pinning feature: The magnetization is small at low applied field $H_{a}$ and increases rapidly while $H_{a}$ reaches a threshold value $H_{\text {th }}$ which corresponds to the field required to exceed the pinning barrier. As the temperature decreases the thresh-
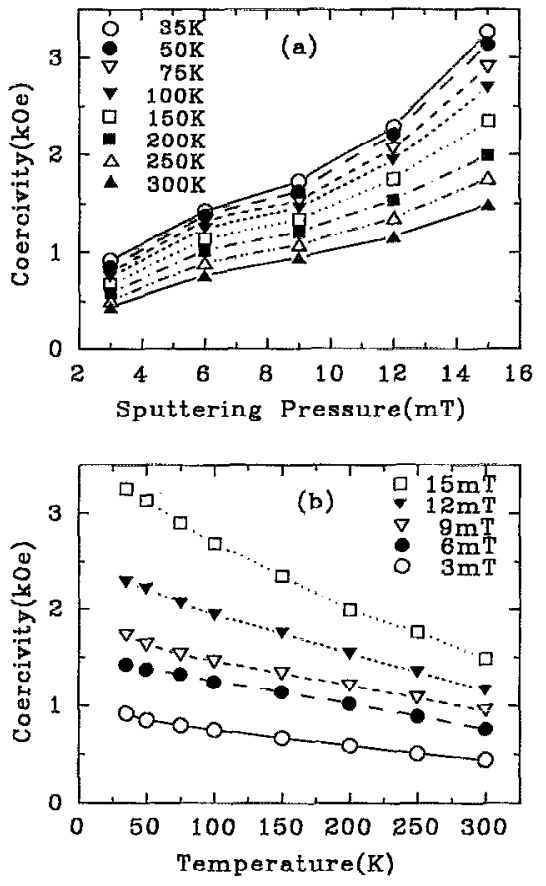

FIG. 4. (a) Sputtering Ar pressure dependence of coercivity at different temperature and $(b)$ temperature dependence of coercivity at different sputtering Ar pressure. 

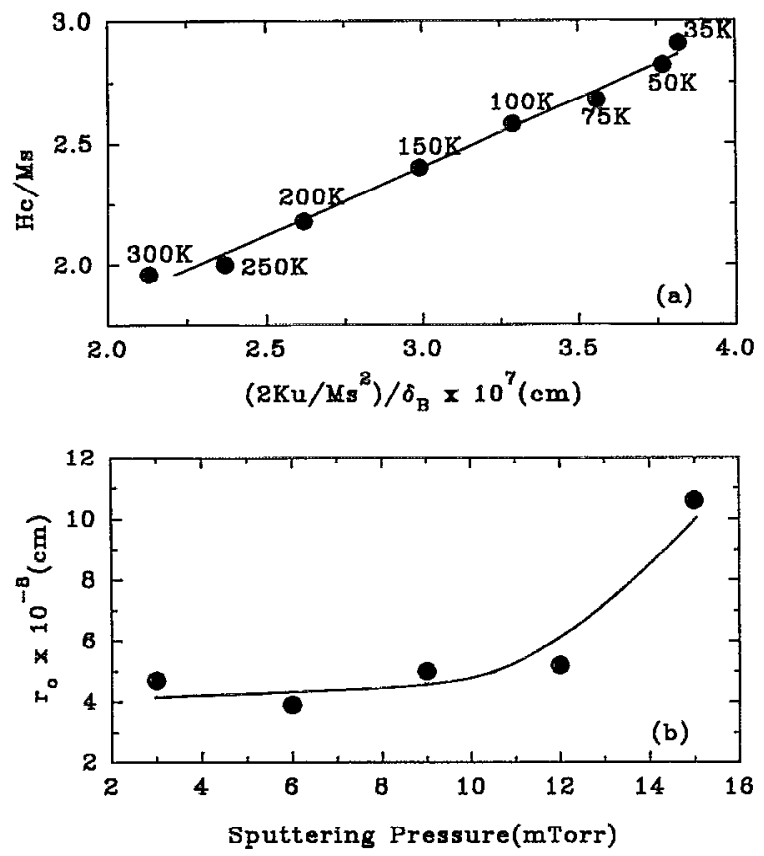

FIG. 5. (a) A linear fitting to the experimental data after $\mathrm{Eq}$. (1) and the $r_{0}$ obtained is $4.7 \AA$. (b) The sputtering Ar pressure dependence of the estimated size of the pinning site.

old field $H_{\text {th }}$ increases because of the decreasing thermal activation energy as predicted by Kirby et al. ${ }^{12}$

Kronmüller's formulas ${ }^{8}$ were used to analyze the coercivity mechanism in more detail. If wall pinning is the dominant mechanism, the coercivity $H_{c}(T)$ is given by

$H_{c}(T)=\kappa\left(r_{0} / \delta_{B}\right)\left(2 K_{u} / M_{s}\right)-N_{\mathrm{eff}} M_{s}$ for $r_{0} \ll \delta_{B}$

and

$H_{c}(T)=\kappa^{\prime}\left(\delta_{B} / r_{0}\right)\left(2 K_{u} / M_{s}\right)-N_{\text {eff }} M_{s}$ for $r_{0} \gg \delta_{B}$,

where $\kappa$ and $\kappa^{\prime}$ are both related to the exchange coupling constants and the anisotropy constants, $r_{0}$ is the size of the pinning site, and $N_{\text {eff }}$ is a demagnetization factor. The wall width $\delta_{B}$ is given by $\pi(A / K)^{1 / 2}$, where $A$ and $K$ are exchange constant and anisotropy, ${ }^{13}$ respectively.

Figure 5 is an example of the fitting curve based on Eq. (1) for the sample prepared at 3 mTorr Ar pressure. Similar fittings for all samples $\left(P_{\mathrm{Ar}}=6,9,12\right.$, and $15 \mathrm{mTorr}$ ) have been performed. The fact that the $\left[H_{c} / M_{s},\left(2 K_{u} / M_{s}^{2}\right) / \delta_{B}\right]$ experimental points measured at different temperatures are on a straight line implies that the domain-wall pinning is the dominant mechanism.
From the fits we could estimate the size of the pinning sites for each sample. The estimated sizes are 4.7, 3.9, 5.0, 5.2, and $10.6 \AA$ for the samples prepared at $P_{\mathrm{Ar}}=3,6,9,12$, and 15 mTorr, respectively [see Fig. 5(b)]. The estimated values show that the size of the pinning site increases with increasing sputtering pressure. Equation (1) also tells us that $H_{c}(T)$ depends on the $r_{0} K_{u}$ product. Although $K_{u}$ decreases with increasing $P_{\mathrm{Ar}}$ for $P_{\mathrm{Ar}}>12 \mathrm{mTorr}$ (as shown in Fig. 3), $H_{c}$ still increases with increasing $P_{\mathrm{Ar}}$ for $P_{\mathrm{Ar}}>12 \mathrm{mTorr}$ [as shown in Fig. 4(a)] because $r_{0}$ increases, and we have pointed out this feature earlier.

\section{CONCLUSIONS}

The variation of the anisotropy and coercivity as a function of temperatures is closely related to the polarization of the Pd atoms at the interfaces and the film morphology which was controlled by the sputtering Ar pressure. The dominant mechanism for the coercivity is the wall pinning and the size of the pinning sites increase with increasing the sputtering pressure.

\section{ACKNOWLEDGMENTS}

We gratefully acknowledge financial support from NSF under Grant No. DMR-9222976. We thank A. Runge for assistance and helpful discussions.

${ }^{1}$ P. F. Carcia, A. D. Meinhaldt, and A. Suna, Appl. Phys. Lett. 47, 78 (1985).

${ }^{2}$ N. Engel, C. D. England, R. A. Van Leeuwen, M. H. Wiedman, and C. M. Falco, Phys. Rev. Lett. 67, 1910 (1991).

${ }^{3}$ F. J. A. den Broeoler, H. C. Donkersloot, H. J. G. Draaisma, and J. M. de Jonge, J. Appl. Phys. 61, 437 (1987).

${ }^{4}$ S. Hashimoto, Y. Ochiai, and K. Aso, J. Appl. Phys. 66, 4909 (1989).

${ }^{5}$ P. de Haan, Q. Meng, T. Katayama, and J. C. Lodder, J. Magn. Magn. Mater. 113, 29 (1992).

${ }^{6}$ S. C. Shin, J. H. Kim, and D. H. Ahn, J. Appl. Phys. 69, 5664 (1991).

${ }^{7}$ P. He, Z. S. Shan, J. A. Woollam, and D. J. Sellmyer, J. Appl. Phys. 73, 5954 (1993).

${ }^{8}$ H. Kronmüller, Phys. Status Solidi B 144, 385 (1987); H. Kronmuller, K. D. Durst, and M. Sagawa, J. Magn. Magn. Mater. 74, 291 (1988).

${ }^{9}$ R. M. Bozorth, P. A. Wolff, D. D. Davis, V. B. Compton, and J. H. Wernick, Phys. Rev. 122, 1157 (1961).

${ }^{10}$ Z. S. Shan, P. He, C. Moore, J. Woollam, and D. J. Sellmyer, J. Appl. Phys. 73, 6057 (1993).

${ }^{11}$ R. H. Victora and J. M. MacLaren, Phys. Rev. B 47, 11583 (1993).

${ }^{12}$ R. D. Kirby, J. X. Shen, R. J. Hardy, and D. J. Sellmyer, Phys. Rev. B 49, 10810 (1994).

${ }^{13}$ T. Suzuki, H. Notarys, D. C. Dobbertin, C. J. Lin, D. Weller, D. C. Miller, and G. Gorman, IEEE Trans. Magn. MAG-28, 2754 (1992). 